\title{
Impact of corticosteroid therapy on the outcomes of hepatocellular carcinoma treated with immune checkpoint inhibitor therapy
}

\author{
David J Pinato (D) , ${ }^{1}$ Ahmed Kaseb, ${ }^{2}$ Yinghong Wang (D) , ${ }^{3}$ Anwaar Saeed, ${ }^{4}$ \\ David Szafron, ${ }^{2}$ Tomi Jun, ${ }^{5}$ Sirish Dharmapuri, ${ }^{5}$ Abdul Rafeh Naqash, ${ }^{6}$ \\ Mahvish Muzaffar, ${ }^{7}$ Musharraf Navaid, ${ }^{7}$ Uqba Khan, ${ }^{8}$ ChiehJu Lee, ${ }^{9}$ \\ Anushi Bulumulle, ${ }^{10}$ Bo Yu, ${ }^{8}$ Sonal Paul, ${ }^{8}$ Petros Fessas (1) , ${ }^{11}$ Neil Nimkar, ${ }^{8}$ \\ Dominik Bettinger, ${ }^{12}$ Hannah Hildebrand, ${ }^{4}$ Tiziana Pressiani, ${ }^{13}$ Yehia I Abugabal, ${ }^{2}$ \\ Nicola Personeni, ${ }^{13,14}$ Yi-Hsiang Huang (D) , ${ }^{9}$ Jingky Lozano-Kuehne, ${ }^{1}$ \\ Lorenza Rimassa, ${ }^{13,14}$ Celina Ang, ${ }^{5}$ Thomas U Marron ${ }^{5}$
}

To cite: Pinato DJ, Kaseb A, Wang $\mathrm{Y}$, et al. Impact of corticosteroid therapy on the outcomes of hepatocellular carcinoma treated with immune checkpoint inhibitor therapy. Journal for ImmunoTherapy of Cancer 2020;8:e000726. doi:10.1136/jitc-2020-000726

- Additional material is published online only. To view, please visit the journal online (http://dx.doi.org/10.1136/jitc2020-000726).

Accepted 02 September 2020

Check for updates

(C) Author(s) (or their employer(s)) 2020. Re-use permitted under CC BY. Published by BMJ.

For numbered affiliations see end of article.

Correspondence to

Dr David J Pinato;

david.pinato@imperial.ac.uk

\section{ABSTRACT}

The impact of corticosteroid therapy (CT) on efficacy of immune checkpoint inhibitors (ICI) is undefined in hepatocellular carcinoma (HCC). We evaluated whether CT administered at baseline (bCT) or concurrently with ICl (cCT) influences overall (OS), progression-free survival (PFS) and overall response rates (ORR) in 341 patients collected across 3 continents. Of 304 eligible patients, 78 $(26 \%)$ received $\geq 10 \mathrm{mg}$ prednisone equivalent daily either as bCT $(n=14,5 \%)$ or CCT ( $n=64,21 \%)$. Indications for CT included procedure/prophylaxis $(n=37,47 \%)$, management of immune-related adverse event $(n=27,35 \%)$, cancerrelated symptoms $(n=8,10 \%)$ or comorbidities $(n=6,8 \%)$. Neither overall CT, bCT nor cCT predicted for worse OS, PFS nor ORR in univariable and multivariable analyses $(p>0.05)$. CT for cancer-related indications predicted for shorter PFS $(p<0.001)$ and was associated with refractoriness to $\mathrm{ICl}(75 \%$ vs $33 \%, \mathrm{p}=0.05)$ compared with cancer-unrelated indications. This is the first study to demonstrate that neither bCT nor CCT influence response and $\mathrm{OS}$ following $\mathrm{ICl}$ in $\mathrm{HCC}$. Worse outcomes in CT recipients for cancer-related indications appear driven by the poor prognosis associated with symptomatic HCC.

\section{INTRODUCTION}

Hepatocellular carcinoma (HCC) is at the focus of intense research efforts for the development of immune checkpoint inhibitors (ICI). ${ }^{1}$ Targeted inhibition of programmed cell death (PD-1) receptor/ligand (PD-1/ PD-L1) interaction results in measurable anti-tumor responses in a fraction of patients with advanced HCC, a finding that led to the breakthrough approval of nivolumab ${ }^{2}$ and pembrolizumab ${ }^{3}$ by the Food and Drug Administration in light of the results of small, single-arm open-label studies. However, evidence of initial activity has not translated into statistically significant survival benefit in randomized controlled studies of anti-PD-1 monotherapy, a finding that has instigated the clinical development of immunotherapy combinations with anti-cytotoxic T-lymphocyte antigen 4 (CTLA-4) inhibitors ${ }^{4}$ and anti-angiogenics to further enhance the antitumor immunity. ${ }^{5}$

Evolving experience in the use of ICI suggests iatrogenic factors as important contributors in shaping clinical responses to immunotherapy. ${ }^{67}$ Corticosteroid therapy (CT) is often indicated in the management of cancer-related symptoms such as cachexia, anorexia, central nervous system edema or pain $^{8}$ and is recommended by guidelines as first-line therapy for most immune-related adverse events (irAEs). ${ }^{9}$ CT exerts T-cell suppressive properties by reducing the proliferative potential of naïve $\mathrm{T}$ cells ${ }^{10}$ and stimulate regulatory T-cell development. ${ }^{11}$

Immunosuppressive CT may therefore adversely influence outcome in patients receiving $\mathrm{ICI}^{12}$ and in fact patients receiving chronic steroid therapy have been for this reason excluded from clinical trials of ICI. The effect of CT either at the moment of ICI commencement or during the course of ICI treatment remains undefined in $\mathrm{HCC}^{13}$ a point of greater consequence in a disease where ICI monotherapy has struggled to demonstrate evidence of sustained clinical benefit. In this global observational study, we sought to document prevalence of and indications for CT use in patients with HCC being treated with ICI, and to examine the relationship between CT exposure and outcome. 


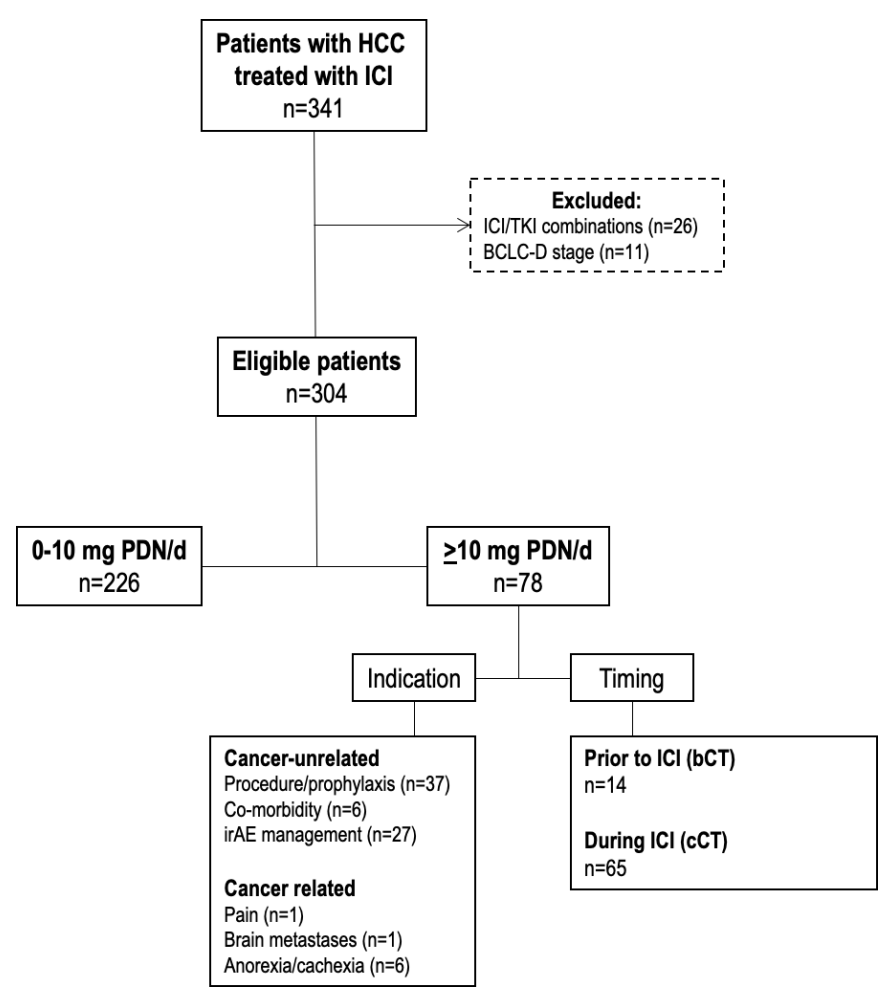

Figure 1 Study diagram of 341 patients with hepatocellular carcinoma (HCC) treated with immune checkpoint inhibitors (ICl). BCLC-D, Barcelona Clinic Liver Cancer stage D; bCT, baseline corticosteroid therapy; cCT, concomitant corticosteroid therapy; irAE, immune-related adverse event; PDN, Prednisolone.; TKI, Tyrosine Kinase Inhibitors.

\section{PATIENTS AND METHODS}

We established a global dataset of 341 patients with HCC treated with ICI between 2016 and 2019 in nine tertiary referral centers in the USA $(n=226)$, Europe $(n=68)$ and Taiwan $(n=47)$ (online supplemental table S1). Patients with a histological or radiologic diagnosis of HCC based on European Society for the Study of the Liver criteria ${ }^{14}$ and undergoing treatment with checkpoint inhibitors were identified from Oncology Pharmacy electronic records and entered into a prospectively maintained database. In total, we excluded 26 patients who were treated with PD-1/PD-L1 inhibitors in combination with kinase inhibitors and a further 11 patients that classified within Barcelona Clinic Liver Cancer (BCLC) stage D due to performance status 3 and/or Child-Pugh C cirrhosis. This resulted in 304 patients eligible for primary analyses (figure 1).

Evaluation of corticosteroid exposure was defined based on timing of administration in line with published literature on the topic. ${ }^{12}$ Patients were defined as receiving CT at baseline (bCT) if administered $\geq 10 \mathrm{mg}$ of prednisone (or equivalent) for $\geq 24$ hours within 30 days prior to ICI. Concomitant corticosteroid therapy (cCT) was defined as stated previously from the day of commencement of ICI until permanent cessation of immunotherapy.

Clinicopathologic variables including overall survival (OS) and progression-free survival (PFS) were derived from electronic medical records. OS was calculated from the date of ICI commencement until last follow-up or patients' death. Response to ICI was evaluated according to RECIST criteria (version 1.1) and best responses to ICI were recorded for each evaluable patient. Electronic medical records were reviewed to identify prescription of oral or intravenous corticosteroid therapy (CT).

\section{STATISTICAL ANALYSIS}

Patient characteristics are summarized as means or medians as appropriate. We conducted analysis of proportions across groups using Pearson $\chi^{2}$ or Fisher's exact tests. We represented group-specific OS and PFS using the Kaplan-Meier curve method and formally evaluated the difference in median survival times between prespecified groups using the log-rank test. We performed univariable and multivariable analyses of survival using Cox regression models to evaluate the impact of CT on patients' OS and PFS. To avoid collinearity bias, we evaluated features relating to corticosteroid therapy including overall CT exposure, timing and indication for CT therapy in separate multivariable models as outlined before. ${ }^{15}$ Post-landmark analysis was done in the subset of patients who received corticosteroid (prednisone $>10 \mathrm{mg}$ ) during ICI treatment. This analysis compared the PFS and OS in patients with/without response with the ICI treatment with cortiscosteroid (CT). Kaplan-Meier analysis was used to estimate the PFS and OS from the time of ICI initiation in patients with $(\mathrm{CR}+\mathrm{PR})$ and without $(\mathrm{SD}+\mathrm{PD})$ response at 3, 6, 9 and 12 months. Patients who were not evaluable for response at a timepoint were excluded.

All statistical analyses were performed using SPSS V.25.0 and Stata V.16.1 (StataCorp LLC, College Station, TX, USA), and GraphPad Prism (GraphPad Software, La Jolla, CA, USA) with all estimates being reported with corresponding $95 \%$ CIs and a two-tailed level of significance of $\mathrm{p}<0.05$.

\section{RESULTS}

\section{Patients' demographics and treatment characteristics}

Out of 304 eligible patients in the study, the majority were cirrhotic $(n=217,71 \%)$ due to hepatitis $\mathrm{C}$ infection $(n=119,39 \%)$. All patients had measurable disease based on RECIST 1.1 criteria at ICI commencement. Baseline tumor staging according to Barcelona Clinic Liver Cancer (BCLC) algorithm showed most patients to qualify criteria for stage $\mathrm{C}$ disease $(\mathrm{n}=230,76 \%)$.

In total, 273 patients $(90 \%)$ had received prior therapy for HCC and 179 (59\%) were sorafenib experienced. During the observation period, the vast majority of patients $(n=279,92 \%)$ received single-agent anti-PD $(L)-1$ ICI, whereas 25 received combined PD-1/CTLA-4 ICI $(8 \%)$.

Evaluation of radiologic response to treatment based on RECIST 1.1 criteria (investigator assessed) demonstrated an ORR of $20 \%$, with 23 complete $(7.5 \%)$ and 38 partial 
responses $(12.5 \%)$. Ninety-nine patients $(33 \%)$ achieved disease stabilization, whereas 127 (42\%) were ICI refractory, defined as progressive disease at first imaging reassessment. After a median follow-up of 10.3 months $(95 \%$ CI 10.6 to 13 months), 153 patients had died (51\%) and all but $72(24 \%)$ had discontinued ICI therapy, mostly due to disease progression $(\mathrm{n}=144,48 \%)$. In total, 113 patients $(37 \%)$ experienced at least one treatment-related adverse event (AE), 57 of which $(18 \%)$ were of grade $>2$ according to Common Terminology Criteria for Adverse Events (version 5.0). Permanent discontinuations due to unacceptable toxicity occurred in 11 patients $(4 \%)$. Median OS from ICI commencement was 12.3 months (95\% CI 9.8 to 15.4 months), median ICI duration was 3.7 months (IQR 6) and median PFS was 6 months (95\% CI 4.6 to 8.2 months).

\section{Corticosteroid therapy}

CT was prescribed to 78 patients (26\%), defined as bCT in $14(5 \%)$ and cCT in $64(20 \%)$. Two patients $(1 \%)$ received CT prior to and during ICI: one patient who received CT for palliation of cancer-related symptoms was ascribed to the bCT group. A second patient who received CT as a single course 7 days prior to ICI for intravenous iron infusion and three subsequent courses during ICI was ascribed to the cCT group. Timing of corticosteroid exposure was not associated with HCC stage, liver function and performance status (table 1). Neither bCT $(p=1.0)$ nor cCT $(p=0.62)$ were associated with line of therapy. The most frequent indication for bCT was periprocedural/prophylactic therapy $(n=12,73 \%)$ in patients who received loco-regional therapy within 30 days from ICI initiation $(n=9)$ or who required CT cover for contrast allergy during imaging studies $(n=3)$. Conversely, irAE management was the most frequent indication for cCT $(\mathrm{n}=27,44 \%, \mathrm{p}=0.003$; online supplemental table S2). Median duration of CT was 4 days (IQR 13) and median daily dose of prednisone equivalent was $50 \mathrm{mg}$ (IQR 30). CT duration was longer in the cCT compared with bCT group (median 6 (IQR 13) vs 2 (IQR 2) days, $\mathrm{p}=0.006$ ), whereas daily prednisone dose was comparable (median 50 (IQR 40) vs 50 (IQR 22.5) $\mathrm{mg} /$ day, $\mathrm{p}=0.09$; online supplemental tables S3-4).

\section{Impact of CT on clinical outcomes from ICI}

The median OS of patients exposed to $\geq 10 \mathrm{mg}$ of prednisone at any point throughout the study was 12.8 months (95\% CI 9 to NR) and not different from the 12.2 months ( $95 \%$ CI 9.7 to $16.1, \mathrm{p}=0.50$ ) observed for patients who received 0 to $<10 \mathrm{mg}$. Median PFS (8.2 months, $95 \%$ CI 5.5 to 12.5 vs 4.8 months, $95 \%$ CI 4.0 to 7.5 months, $\mathrm{p}=0.25$ ) and ORR (21.3\% vs $21.1 \%, \mathrm{p}=0.96$ ) were also similar between groups. When compared with bCT-unexposed (median OS 12.2, 95\% CI 8.7 to 15.6) or cCT-unexposed patients (median OS 11.7, 95\% CI 9.4 to 13.9), neither bCT (median OS 10.4, 95\% CI 7.5 to 13.3, $\mathrm{p}=0.53$ ) nor cCT exposure (median OS 16.1, 95\% CI 8.8 to 22.5) was indicative of worse OS (figure 2A-C). No difference in median PFS was observed across bCT (6.7 months, 95\% CI 1.0 to 13.0 months vs 5.8 months, $95 \%$ CI 4.0 to 7.6, $\mathrm{p}=0.36)$, cCT (8.1 months, $95 \%$ CI 5.5 to 10.7 months vs 4.9 months, $95 \%$ CI 3.3 to $6.5, \mathrm{p}=0.42$; figure $2 \mathrm{E}, \mathrm{F}$ ).

Next, we evaluated the distribution of radiologic responses across bCT, cCT or steroid-unexposed patients and found no significant difference across groups $(\mathrm{p}=0.62$, figure 3A). Patients on CT for palliation of cancer-related symptoms were more likely to be ICI refractory (figure 3B, $\mathrm{p}=0.05)$, and have shorter median PFS (1.6 months, $95 \%$ CI 0.4 to 2.8 vs 8.8 months, $95 \%$ CI 4.2 to 13.5 , p<0.01) and OS (4.9 months, $95 \%$ CI 0.5 to 14.1 vs 15.4 months, $95 \%$ CI 7.0 to $18.6, \mathrm{p}=0.05$, online supplemental figure 1 A-C) compared with cancer-unrelated indications.

Univariable analyses of survival revealed no difference in OS based on median duration of treatment $(\mathrm{p}=0.15)$ and median daily corticosteroid dose $(\mathrm{p}=0.75$, online supplemental figure S2). In independent univariable and multivariable Cox regression models, neither bCT nor cCT predicted for OS or PFS ( $\mathrm{p}>0.05$, online supplemental tables S5-8). Analyses of OS and PFS in patients with Child-Pugh A cirrhosis were consistent with estimates observed in the whole patient population (online supplemental figures S3,4). We further tested the relationship between CT exposure and response, OS and PFS using post-landmark analysis to account for the time dependence of CT administration. In the first 3 months, 19 $(1.6 \%)$ of the 62 evaluable patients with CT exposure during ICI had at least one course of corticosteroids. The median PFS and OS in these patients were 4.6 months (95\% CI 1.4 to 9) and 7.5 months (2.5 to NR), respectively. The median PFS and OS in the subgroup of patients with $\mathrm{CR}+\mathrm{PR}$ response were not reached. Succeeding timepoints (months 6, 9 and 12) showed longer PFS and OS durations for cCT compared with those without CT exposure (ie, 0 to $10 \mathrm{mg}$ prednisone) or bCT (online supplemental table S9). When evaluating patients exposed to CT at any time point during ICI, PFS was 8.2 months (95\% CI 5.6 to 12.5 ) while OS was 16.1 months (95\% CI 8.9 to NR). Comparison of PFS and OS across the three groups of CT exposure did not show significant results $(\mathrm{p}>0.05)$.

\section{DISCUSSION}

We document for the first time that corticosteroid use alongside ICI is safe in patients with HCC, mirroring evidence in other indications. ${ }^{16} 17$ This is particularly reassuring for patients requiring cCT for irAE management, who, despite receiving higher doses of steroids for longer periods compared with other indications, had similar outcomes compared with steroid-unexposed patients. The low prevalence of bCT $(5 \%)$, not predictive of outcome in our study, is perhaps unsurprising given that, unlike advanced lung cancer, unresectable HCC is a largely asymptomatic diagnosis and intracranial spread is rare. ${ }^{18}$ Interestingly, only patients with HCC receiving palliative CT had worse ORR, PFS and OS. This lends 
Table 1 Clinicopathologic features of the patient population

\section{Corticosteroid exposure}

\begin{tabular}{|c|c|c|c|c|c|}
\hline Characteristic & $\begin{array}{l}\text { All patients } \\
n=304(\%)\end{array}$ & $\begin{array}{l}\text { Prednisone } 0 \text { to } \\
<10 \mathrm{mg} \mathrm{n}=226 \\
(\%)\end{array}$ & $\begin{array}{l}\text { Prednisone }>10 \text { mg prior } \\
\text { to } \mathrm{ICI} n=14(\%)\end{array}$ & $\begin{array}{l}\text { Prednisone }>10 \mathrm{mg} \\
\text { during } \mathrm{ICI} \mathrm{n}=64(\%)\end{array}$ & P value* \\
\hline Gender & & & & & 0.68 \\
\hline Female & $69(23)$ & $54(24)$ & $3(21)$ & $12(18)$ & \\
\hline Cirrhosis & & & & & 0.23 \\
\hline Absent & $87(29)$ & $69(31)$ & $5(36)$ & $13(20)$ & \\
\hline Unknown & $1(0.3)$ & $1(0.4)$ & $0(0)$ & $0(0)$ & \\
\hline Etiology of chronic liver disease & & & & & 0.85 \\
\hline Viral & $196(65)$ & $144(64)$ & $10(71)$ & $42(66)$ & \\
\hline Non-viral & $106(34)$ & $80(35)$ & $4(29)$ & $22(34)$ & \\
\hline $\mathrm{B}$ & $79(26)$ & $56(25)$ & $5(36)$ & $18(28)$ & \\
\hline Barcelona Clinic Liver Cancer stage & & & & & 0.35 \\
\hline$A-B$ & $74(24)$ & $51(23)$ & $3(21)$ & $20(31)$ & \\
\hline $\mathrm{C}$ & $230(76)$ & $175(77)$ & $11(79)$ & $44(69)$ & \\
\hline $\begin{array}{l}\text { Maximum diameter of largest lesion } \\
(\mathrm{cm})\end{array}$ & & & & & 0.96 \\
\hline Median (IQR) & $5.5(5.5)$ & $5.5(6)$ & $5(-)$ & $5(4.5)$ & \\
\hline $\mathrm{n}$ & 202 & 166 & 1 & 35 & \\
\hline Metastatic sites & & & & & 0.81 \\
\hline Immunotherapy treatment & & & & & 0.1 \\
\hline Monotherapy & $279(92)$ & $204(82)$ & $14(100)$ & $61(91)$ & \\
\hline Combination & $25(8)$ & $22(18)$ & $0(0)$ & $3(9)$ & \\
\hline ECOG Performance Status & & & & & 0.46 \\
\hline $0-1$ & $197(65)$ & $162(72)$ & $1(7)$ & $34(53)$ & \\
\hline$\geq 2$ & $13(4)$ & $9(4)$ & $0(0)$ & $4(6)$ & \\
\hline Unknown & $94(31)$ & $55(24)$ & $13(93)$ & $26(41)$ & \\
\hline Alfa-fetoprotein & & & & & 0.42 \\
\hline$<400 \mathrm{ng} / \mathrm{mL}$ & $178(59)$ & $129(57)$ & $10(71)$ & $39(61)$ & \\
\hline$>400 \mathrm{ng} / \mathrm{mL}$ & $118(39)$ & $93(41)$ & $4(29)$ & $21(33)$ & \\
\hline Unknown & $8(3)$ & $4(2)$ & $0(0)$ & $4(6)$ & \\
\hline Albumin & & & & & 0.25 \\
\hline Median (IQR) & $36(9)$ & $36(8)$ & $33(10)$ & $36(10)$ & \\
\hline $\mathrm{n}$ & 298 & 220 & 14 & 64 & \\
\hline Bilirubin & & & & & 0.64 \\
\hline Median (IQR) & $14(12)$ & $14(12)$ & 14.5 (12) & $15(10)$ & \\
\hline $\mathrm{n}$ & 302 & 224 & 14 & 64 & \\
\hline
\end{tabular}

Continued 
Table 1 Continued

\begin{tabular}{|c|c|c|c|c|c|}
\hline \multirow[b]{2}{*}{ Characteristic } & \multirow[b]{2}{*}{$\begin{array}{l}\text { All patients } \\
\mathrm{n}=304(\%)\end{array}$} & \multicolumn{3}{|c|}{ Corticosteroid exposure } & \multirow[b]{2}{*}{ P value* } \\
\hline & & $\begin{array}{l}\text { Prednisone } 0 \text { to } \\
<10 \mathrm{mg} \mathrm{n}=226 \\
(\%)\end{array}$ & $\begin{array}{l}\text { Prednisone }>10 \mathrm{mg} \text { prior } \\
\text { to } \mathrm{ICI} n=14(\%)\end{array}$ & $\begin{array}{l}\text { Prednisone }>10 \mathrm{mg} \\
\text { during } \mathrm{ICI} n=64(\%)\end{array}$ & \\
\hline Platelet count & & & & & 0.58 \\
\hline Median (IQR) & $160(119)$ & $160(120)$ & $100(-)$ & $162(92)$ & \\
\hline $\mathrm{n}$ & 210 & 171 & 1 & 38 & \\
\hline
\end{tabular}

"Excludes the "Unknown" category in the statistical test.

ECOG, Eastern Cooperative Oncology Group; HCC, hepatocellular carcinoma; ICI, immune checkpoint inhibitors.

credence to the view that CT may correlate with prognosis by association with poor prognostic features (ie, cancer-related symptoms secondary to symptomatic or rapidly progressive disease) rather than blunting of ICI responsiveness. ${ }^{19}$

While limited by small sample size and lack of correlative analyses on peripheral immune cell responses following steroid treatment, the multi-center design of our study ensures adequate representation of the various etiologies of HCC and attempts to control for the diversity in clinical practice including corticosteroid prescribing. Given this study enrolled patients treated with ICI as part of routine clinical practice, our sample included patients with Child-Pugh B cirrhosis, largely excluded from clinical trials of ICI in HCC. While impaired liver function is a key prognostic determinant in $\mathrm{HCC},{ }^{20}$ this was unrelated to the provision of CT and supplementary analyses inclusive of Child-Pugh A patients only were concordant with main study outcomes (online supplemental figures S3-4). We could not ascertain the relationship between
CT and comorbidities other than liver dysfunction, an aspect worth exploring in prospective studies.

To conclude, there is no sufficient evidence to suggest that CT exposure either prior to or during ICI therapy is associated with OS and PFS in patients with HCC; CT exposure does not associate with key clinicopathologic traits of HCC including stage, liver function, alphafetoprotein levels and line of therapy. CT for palliative indications identified patients with poorer response and survival from ICI and traces an interesting parallelism with evolving experience in lung cancer where association with adverse features rather than causality has emerged as a likely explanation of the detrimental role of $\mathrm{CT} .{ }^{19}$ While mechanistic studies on the immune-modulatory effects of CT in ICI recipients are awaited, our clinical data are reassuring in suggesting that CT does not appear to worsen ORR and OS in HCC being treated with ICI. The relationship between CT and outcomes from combination regimens including ICI and anti-angiogenics should be further explored in prospective studies.

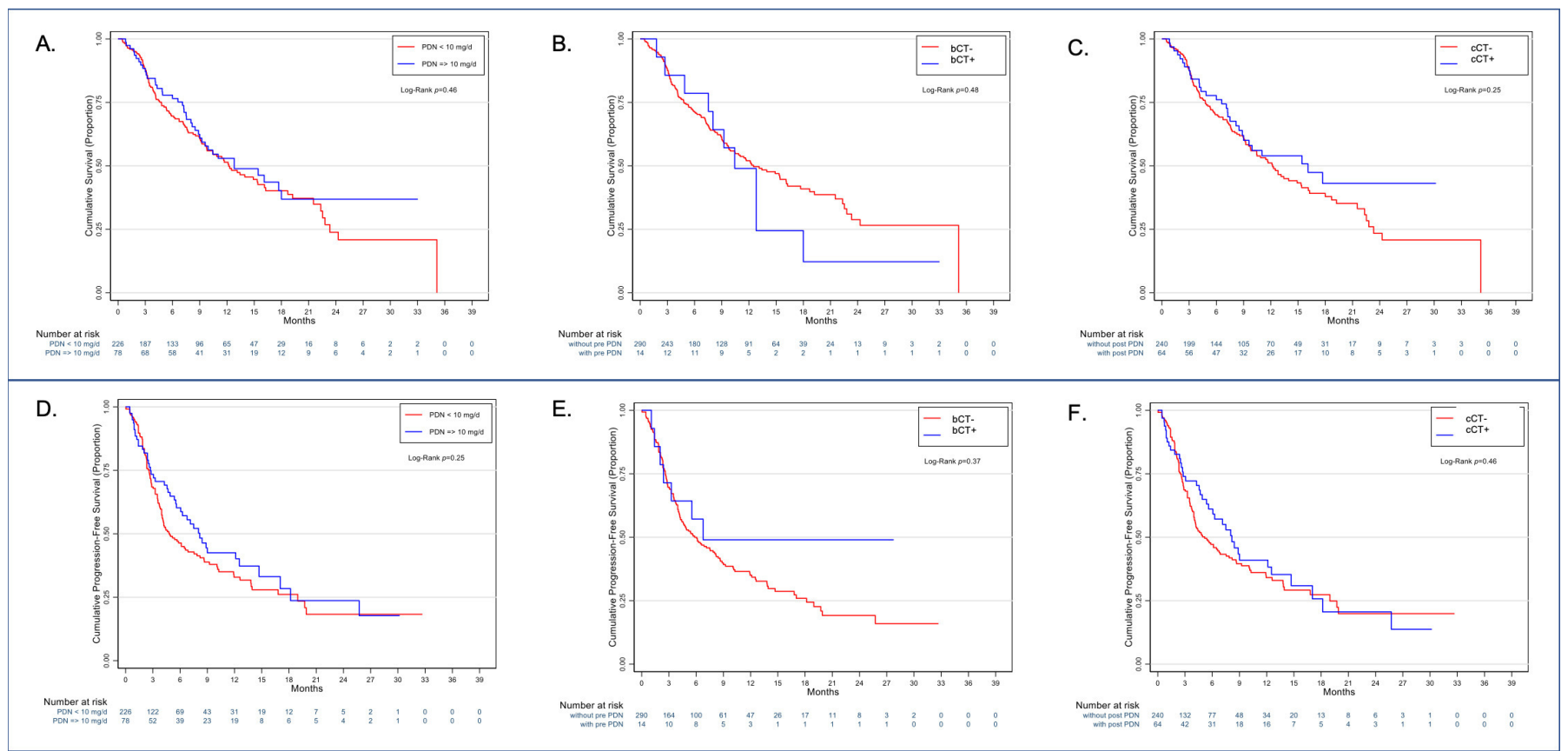

Figure 2 Exposure to corticosteroid therapy (CT, panel A) and timing of CT (panels B, C) do not influence the overall survival and progression-free survival (panels D, E, F) of patients with hepatocellular carcinoma receiving immune checkpoint inhibitors. 


\section{A}

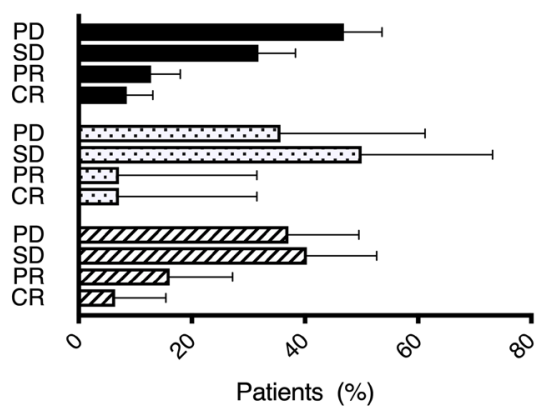

B

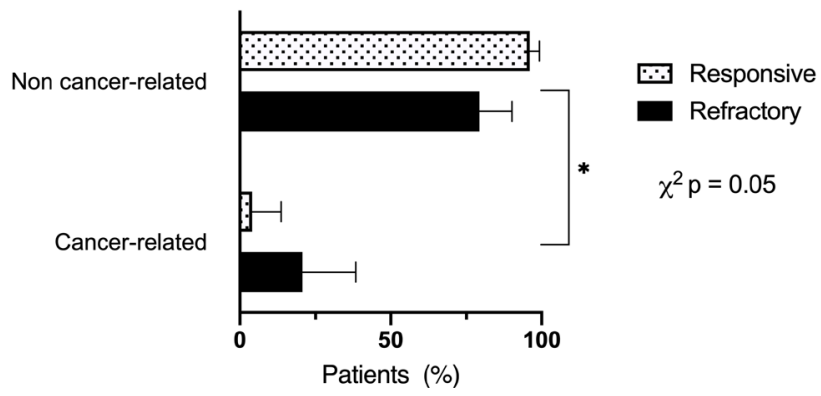

Figure 3 Relationship between timing of corticosteroid therapy $(C T)(A)$, indication of CT (B) and response to immune checkpoint inhibitors (ICI) in hepatocellular carcinoma. Patients were defined as $\mathrm{ICI}$ responsive if they achieved complete response (CR), partial response (PR) or stable disease (SD) $(n=160)$ as best overall response by RECIST version 1.1 criteria. Refractory patients were defined as those achieving progressive disease (PD) as best response to $\mathrm{ICl}(\mathrm{n}=127)$. bCT, baseline corticosteroid therapy; $\mathrm{CCT}$, concomitant corticosteroid therapy.

\section{Author affiliations}

${ }^{1}$ Department of Surgery and Cancer, Imperial College London, London, UK ${ }^{2}$ Department of Gastrointestinal Medical Oncology, The University of Texas MD Anderson Cancer Center, Houston, Texas, USA

${ }^{3}$ Department of Gastroenterology, Hepatology \& Nutrition, University of Texas MD Anderson Cancer Center, Houston, Texas, United States

${ }^{4}$ Division of Medical Oncology, Kansas University Cancer Center, Kansas City, Kansas, USA

${ }^{5}$ Department of Medicine, Division of Hematology/Oncology, Tisch Cancer Institute, Mount Sinai Hospital School of Medicine, New York, New York, USA

${ }^{6}$ National Cancer Institute, Bethesda, Maryland, USA

${ }^{7}$ Division of Hematology/Oncology, East Carolina University, Greenville, North Carolina, USA

${ }^{8}$ Division of Hematology and Oncology, Weill Cornell Medicine/New York Presbyterian Hospital, Ithaca, New York, USA

${ }^{9}$ Division of Gastroenterology and Hepatology, Department of Medicine at Taipei Veterans General Hospital and Institute of Clinical Medicine, National Yang-Ming University, Taipei, Taiwan

${ }^{10}$ Brody School of Medicine at East Carolina University, Greenville, North Carolina, USA

${ }^{11}$ Surgery and Cancer, Imperial College London, London, UK

${ }^{12}$ Department of Medicine II, Faculty of Medicine, Medical Center University of Freiburg, Freiburg, Baden-Württemberg, Germany

${ }^{13}$ Medical Oncology and Hematology Unit, Humanitas Cancer Center, Humanitas Clinical and Research Center, IRCCS, Via Manzoni 56, Rozzano, Milan, Italy
${ }^{14}$ Department of Biomedical Sciences, Humanitas University, Via Rita Levi Montalcini 4, 20090 Pieve Emanuele, Milan, Italy, Rozzano, Lombardia, Italy

Correction notice This article has been corrected since publication. Tiziana Pressiani's affiliation was updated to 'Medical Oncology and Hematology Unit, Humanitas Cancer Center, Humanitas Clinical and Research Center, IRCCS, Via Manzoni 56, Rozzano, Milan, Italy'

Twitter Abdul Rafeh Naqash @thenasheffect and Uqba Khan @uqbakhan

Acknowledgements The authors would like to acknowledge the infrastructure support provided by Imperial Experimental Cancer Medicine Center, Cancer Research UK Imperial Center and the Imperial College Healthcare NHS Trust Tissue Bank.

Contributors Study concept and design: DP. Acquisition of data: AK, YW, AS, DS, SD, TJ, ARN, UK, JL-K, AB, BY, SP, NN, DB, HH, TP, YIA, NP, MM, MN, Y-HH, LR, CA, PF, TM. Analysis and interpretation of data: DP, JL-K, TM. Drafting of the manuscript: DP, TM. Critical revision of the manuscript for important intellectual content: all authors. Statistical analysis: DP, JL-K. Obtained funding: DP. Administrative, technical or material support: DP, AK, YW, AS, ARN, UK, Y-HH, LR, TM. Study supervision: DP, TM.

Funding DB is supported by the Berta-Ottenstein Program, Faculty of Medicine, University of Freiburg. DP is supported by grant funding from the Wellcome Trust Strategic Fund (PS3416).

Competing interests DP received lecture fees from ViiV Healthcare, Bayer Healthcare and travel expenses from BMS and Bayer Healthcare; consulting fees for Mina Therapeutics, EISAI, Roche, Astra Zeneca; received research funding (to institution) from MSD, BMS. DB has received lecture and speaker fees from Bayer Healthcare and the Falk Foundation Germany. LR received lecture fees from AbbVie, Amgen, Eisai, Gilead, Incyte, Ipsen, Lilly, Roche, Sanofi; advisory board/consulting fees from Amgen, ArQule, AstraZeneca, Basilea, Bayer, Celgene, Eisai, Exelixis, Hengrui, Incyte, Ipsen, Lilly, MSD, Nerviano Medical Sciences, Roche, Sanofi; travel expenses from Ipsen; received research funding (to institution) from Agios, ARM0 BioSciences, AstraZeneca, BeiGene, Eisai, Exelixis, Fibrogen, Incyte, Ipsen, Lilly, MSD, Roche. NP received lecture fees from AbbVie and Gilead; travel expenses from ArQule. Y-HH has received advisory board/consulting fees for BMS, MSD, Bayer Healthcare, IPSEN, EISAl, Gilead and Lilly. AS received research funding (to institution) from AstraZeneca, Exelixis, BMS and Clovis; advisory board/consulting fees from BMS, AstraZeneca and Exelixis.

Patient consent for publication Written consent was waived due to the retrospective nature of the study.

Ethics approval Ethical approval to conduct this study was granted by the Imperial College Tissue Bank (Reference Number R16008) under a broader protocol to investigate tissue and clinical predictors of outcome in patients with metastatic malignancies undergoing ICI treatment. Institutional Review Board approval was obtained at every participating center before study initiation. All study-related procedures and data collection were conducted in accordance to the Declaration of Helsinki and in accordance with Good Clinical Practice.

Provenance and peer review Not commissioned; externally peer reviewed.

Data availability statement Data are available under the auspices of the principal investigator on written request.

Open access This is an open access article distributed in accordance with the Creative Commons Attribution 4.0 Unported (CC BY 4.0) license, which permits others to copy, redistribute, remix, transform and build upon this work for any purpose, provided the original work is properly cited, a link to the licence is given, and indication of whether changes were made. See https://creativecommons.org/ licenses/by/4.0/.

\section{ORCID iDs}

David J Pinato http://orcid.org/0000-0002-3529-0103

Yinghong Wang http://orcid.org/0000-0002-5148-6130

Petros Fessas http://orcid.org/0000-0002-7472-8913

Yi-Hsiang Huang http://orcid.org/0000-0001-5241-5425

\section{REFERENCES}

1 Flynn MJ, Sayed AA, Sharma R, et al. Challenges and opportunities in the clinical development of immune checkpoint inhibitors for hepatocellular carcinoma. Hepatology 2019;69:2258-70. 
2 El-Khoueiry AB, Sangro B, Yau T, et al. Nivolumab in patients with advanced hepatocellular carcinoma (CheckMate 040): an open-label, non-comparative, phase 1/2 dose escalation and expansion trial. Lancet 2017;389:2492-502.

3 Zhu AX, Finn RS, Edeline J, et al. Pembrolizumab in patients with advanced hepatocellular carcinoma previously treated with sorafenib (KEYNOTE-224): a non-randomised, open-label phase 2 trial. Lancet Oncol 2018;19:940-52.

4 Yau T, Kang Y-K, Kim T-Y. Nivolumab (NIVO)+ ipilimumab (IPI) combination therapy in patients (pts) with advanced hepatocellular carcinoma (aHCC): Results from CheckMate 040. Am J Clin Oncol 2019.

5 Kudo M. A new era in systemic therapy for hepatocellular carcinoma: atezolizumab plus bevacizumab combination therapy. Liver Cancer 2020;9:119-37.

6 Pinato DJ, Howlett S, Ottaviani D, et al. Association of prior antibiotic treatment with survival and response to immune checkpoint inhibitor therapy in patients with cancer. JAMA Oncol 2019;5:1774.

7 Rossi G, Pezzuto A, Sini C, et al. Concomitant medications during immune checkpoint blockage in cancer patients: novel insights in this emerging clinical scenario. Crit Rev Oncol Hematol 2019;142:26-34.

8 Miller S, McNutt L, McCann M-A, et al. Use of corticosteroids for anorexia in palliative medicine: a systematic review. J Palliat Med 2014; $17: 482-5$.

9 Michot JM, Bigenwald C, Champiat S, et al. Immune-related adverse events with immune checkpoint blockade: a comprehensive review. Eur J Cancer 2016;54:139-48.

10 Giles AJ, Hutchinson M-KND, Sonnemann HM, et al. Dexamethasone-induced immunosuppression: mechanisms and implications for immunotherapy. J Immunother Cancer 2018;6:51

11 Libert C, Dejager L. How steroids steer T cells. Cell Rep 2014;7:938-9.
12 Hussain N, Naeem M, Pinato DJ. Concomitant medications and immune checkpoint inhibitor therapy for cancer: causation or association? Hum Vaccin Immunother 2020:1-7.

13 Arbour KC, Mezquita L, Long N, et al. Impact of baseline steroids on efficacy of programmed cell death-1 and programmed death-ligand 1 blockade in patients with non-small-cell lung cancer. J Clin Oncol 2018;36:2872-8.

14 European Association for the Study of the Liver. Electronic address: easloffice@easloffice.eu, European Association for the Study of the Liver. EASL clinical practice guidelines: management of hepatocellular carcinoma. J Hepatol 2018;69:182-236.

15 Pinato DJ, Sharma R, Allara E, et al. The ALBI grade provides objective hepatic reserve estimation across each BCLC stage of hepatocellular carcinoma. J Hepatol 2017;66:338-46.

16 Santini FC, Rizvi H, Plodkowski AJ, et al. Safety and efficacy of retreating with immunotherapy after immune-related adverse events in patients with NSCLC. Cancer Immunol Res 2018;6:1093-9.

17 Horvat TZ, Adel NG, Dang T-O, et al. Immune-related adverse events, need for systemic immunosuppression, and effects on survival and time to treatment failure in patients with melanoma treated with ipilimumab at Memorial Sloan Kettering Cancer Center. J Clin Oncol 2015;33:3193-8

18 Villanueva A. Hepatocellular carcinoma. N Engl J Med 2019;380:1450-62.

19 Ricciuti B, Dahlberg SE, Adeni A, et al. Immune checkpoint inhibitor outcomes for patients with non-small-cell lung cancer receiving baseline corticosteroids for palliative versus nonpalliative indications. $J$ Clin Oncol 2019:37:1927-34.

20 Pinato DJ, Kaneko T, Saeed A, et al. Immunotherapy in hepatocellular cancer patients with mild to severe liver dysfunction: adjunctive role of the ALBI grade. Cancers 2020;12. doi:10.3390/cancers12071862. [Epub ahead of print: $10 \mathrm{Jul}$ 2020]. 